\title{
Aplicación de herramientas computacionales al estudio morfotaxonómico del género Merremia (Convolvulaceae) en Venezuela
}

\author{
Computational tools employed in morphotaxonomical studies \\ of Merremia (Convolvulaceae) from Venezuela
}

Hernán Ferrer-Pereira ${ }^{1 *}$, Yuribia Vivas-Arroyo ${ }^{1}$, Omaira Hokche $^{1}$, Shingo Nozawa ${ }^{1}$,
Silvia Pérez-Cortéz ${ }^{1}$, Leyda Rodríguez ${ }^{1}$, Julián Mostacero ${ }^{1}$ \& Javier Estrada-Sánchez ${ }^{2}$

\begin{abstract}
Resumen
La implementación de programas computacionales es una alternativa para facilitar la caracterización objetiva de los taxones. En la revisión de Merremia para Venezuela, se empleó el lenguaje y los programas informáticos del Sistema DELTA. Para la definición de la tabla de caracteres/estados se analizaron las descripciones publicadas sobre los taxones registrados para Venezuela y se contrastaron con la variabilidad observada en 129 Unidades Taxonómicas Operacionales (UTOs) (especímenes de los herbarios VEN, MY y PORT, fototipos y descripciones), resultando en una matriz de 209 caracteres cuantitativos y cualitativos. Con el Editor de Delta se codificó la variabilidad morfológica de las 129 UTOs y se obtuvieron matrices de distancias, analizadas con el programa PAST para establecer la delimitación taxonómica mediante los análisis multivariantes de ordenamiento y clasificación. Las descripciones de los UTOs reconocidos como correspondientes a cada taxón en los análisis multivariantes se consolidaron usando INTKEY. Estas últimas fueron reintroducidas en Editor DELTA para elaborar las descripciones en lenguajenatural y las claves, tanto multientrada para INTKEY como dicotómicas. Los resultados obtenidos corroboraron la presencia de 12 especies de Merremia en Venezuela y permitieron el reconocimiento de nuevos caracteres útiles para la diferenciación entre taxones.
\end{abstract}

Palabras clave: análisis de similitud, DELTA, INTKEY, morfología.

\begin{abstract}
The implementation of software is an alternative to help with objective characterization of taxa. In a review of Merremia for Venezuela the language and software of the DELTA system were used. To define the table of characters/states, published descriptions of the taxa recorded in Venezuela were analyzed and compared with the observed variability in 129 Operational Taxonomic Units (OTU's) (VEN, MY, and PORT herbarium specimens, phototypes and taxa descriptions), resulting in a matrix of 209 quantitative and qualitative characters. OTU morphological variability was codified using Delta Editor and distance matrices were obtained with PAST software to establish the taxonomic delimitation using ordering and clustering multivariate analyses. The descriptions of OTUs recognized as belonging to each taxon in the multivariate analyses were consolidated using INTKEY. The latter were reintroduced into DELTA Editor to perform natural language descriptions, and to obtain both dichotomy and multi-input keys for the INTKEY software. The results confirmed the presence of 12 species of Merremia in Venezuela and allowed the recognition of new characters for differentiation among taxa.
\end{abstract}

Key words: DELTA, INTKEY, morphology, similiarity analysis.

\section{Introducción}

La selección de caracteres y sus estados en los estudios de taxonomía clásica debe estar sujeta a la mayor simplicidad posible. Esto se traduce en un incremento del rigor de la sistemática morfológica, disminución de la subjetividad en el proceso de selección de caracteres, en la validación de los criterios de selección y de las propiedades de los tipos de caracteres evaluados (Poe \& Wiens 2000).

\footnotetext{
'Gerencia de Investigación y Desarrollo, Fundación Instituto Botánico de Venezuela “Dr. Tobías Lasser”, Apartado 2156, Caracas 1010-A, Venezuela.

${ }^{2}$ Departamento de Botánica, Escuela de Botánica, Facultad de Ciencias, Universidad de los Andes, Apartado 52, La Hechicera, Mérida, 5212, Venezuela.

*Autor para correspondencia: hferrerp@gmail.com
} 
El uso y procesamiento de gran cantidad de datos a partir de los caracteres que se emplean en sistemática vegetal conducen a un trabajo engorroso de forma manual que podría aumentar la subjetividad del experto y reduce la percepción de la variación morfológica, generando dificultades para la categorización de los taxones evaluados. Una de las posibilidades para resolver estas dificultades consiste en la implementación de programas computacionales como alternativa a la taxonomía vegetal tradicional. El paquete de programas DELTA (DEscription Language for TAxonomy) es una de estas alternativas, siendo estandarizado para desarrollar investigaciones taxonómicas y la obtención de descripciones en lenguaje natural, así como claves interactivas de identificación y matrices de datos multivariantes para análisis filogenéticos y fenéticos (Dallwitz \& Payne 1993; Dallwitz et al. 2008).

Este paquete de programas ha sido utilizado en estudios sistemáticos de gramíneas (Clayton et al. 2010), claves para la identificación de especies y familias de angiospermas (Estrada 1995; Garbiso \& Estrada 2001; Dallwitz et al. 2008). En este sentido, el objetivo de este trabajo consistió en determinar los aportes de las aplicaciones de DELTA para el análisis de un conjunto amplio de datos en corto tiempo, utilizando especímenes del género Merremia Dennst. ex Endl. depositados en los principales herbarios venezolanos.

\section{Materiales y Métodos}

Se revisaron 129 fuentes de datos (fotografías de ejemplares tipo, descripciones originales y especímenes) provenientes de las colecciones de los herbarios VEN, MY y PORT, considerando incluir la variación observada entre las especies de Merremia reportadas para Venezuela. Estos especímenes fueron comparados entre sí para observar diferencias y similitudes de caracteres y luego contrastados con lo señalado en la bibliografía (Pittier et al. 1945; Austin \& Staples 1981, 1983; Austin 1982). Por medio de las observaciones de las características morfológicas de los especímenes y la revisión de literatura (Stefanovic et al. 2002, 2003), se alcanzó el primer paso para identificar las posibles homologías y variaciones entre los caracteres y sus estados definidos para esta investigación.

Para optimizar la definición de los caracteres y sus estados, se revisó la literatura botánica especializada en morfología, se establecieron diversos caracteres siguiendo los criterios y terminología de Radford et al. (1967), Hickey (1973), Dilcher(1974), Payne (1978), Austin (1982) y Weberling (1996). De este modo, se estableció un conjunto de caracteres potenciales que no habían sido estudiados según la literatura consultada y que se esperaba aportaran diferencias entre taxones. Asimismo, se consideró la relevancia de los caracteres y estados reportados previamente.

Una vez que la variación morfológica fue identificada, se creó una matriz de datos utilizando la herramienta Editor DELTA (Dallwitz \& Paine 1993), con la mayor cantidad de información posible a partir de cada pliego, incluyendo el conjunto de caracteres referidos por otros autores, así como los novedosos observados en el desarrollo de la presente investigación. Consecuentemente, los estados de cada carácter fueron establecidos con base en el rango observado para un mismo carácter en los pliegos de todos los taxones potenciales. El perfeccionamiento de la matriz se obtuvo mediante evaluaciones preliminares de los posibles resultados y, según el resultado obtenido y la discusión sostenida por la literatura botánica, se eliminaron, modificaron y/o agregaron caracteres y estados a fin de optimizar la representación natural de la morfología estudiada y mantener la ilación más acorde con la descripción en lenguaje natural.

Dada la revisión y ajuste de los datos en Editor DELTA, se obtuvieron la matriz de distancia para análisis fenéticos de datos multivariantes y la clave interactiva de los pliegos "Intkey_pliegos" (Dallwitz et al. 2008) para proceder a la definición de los taxones en función de los agrupamientos formados.

Cada uno de los especímenes fue ingresado en la matriz del Editor DELTA y etiquetado únicamente con el apellido y número de los colectores además del herbario de depósito de cada pliego. Separadamente, en una base de datos de Access Microdoft Office 2003, se ingresaron los datos de localidad y ecología de los especímenes seleccionados y se agregaron dos campos para colocar etiquetas cortas (con menos de ocho caracteres) en cada pliego, por ejemplo atu_3, y otro para registrar el estado fenológico de la muestra. Posteriormente, las etiquetas cortas se utilizaron para identificar los pliegos en el análisis fenético.

La matriz de distancia obtenida con el Editor DELTA fue editada en una hoja de cálculo para producir una matriz rectangular, la cual se introdujo en PAST (Hammer et al. 2001) para obtener un análisis fenético, a partir de datos multivariantes, y se determinaron las afinidades entre los especímenes 
evaluados por medio de un dendrograma. Se obtuvieron dos dendrogramas por medio de los métodos jerárquicos de Ward (1963) y UPGMA (Sokal \& Sneath 1973).

Los grupos obtenidos en los dendrogramas fueron comparados con las determinaciones taxonómicas actuales. Los pliegos que mostraron agrupamientos incongruentes fueron revisados nuevamente para confirmar su determinación y se corrigieron aquellas erradas, conjuntamente con sus etiquetas cortas respectivas en la base de datos. Se ejecutó el análisis con los datos corregidos para obtener un dendrograma final. Cabe destacar, que se observaron algunas incongruencias de afinidad causadas por la información incompleta de los pliegos utilizados y posibles errores humanos durante el registro de los datos en la matriz del Editor DELTA, que pueden ser justificables y excluidas del análisis.

Por medio del archivo "Intkey_pliegos", se seleccionaron los especímenes que mostraban el mismo agrupamiento para definir la variación del taxón a través de la acción "Summary". Este producto del Intkey_pliegos fue obtenido para los grupos taxonómicos observados y se colocaron en un archivo de texto sin formato (.txt) siguiendo las directrices del programa Editor para crear una nueva matriz de datos con el Editor DELTA, únicamente para taxones, conservando la misma tabla de caracteres y sus estados y los comandos de especificaciones utilizados para la matriz de pliegos.

Con esta nueva matriz definida sólo para taxones, se obtuvieron las descripciones en lenguaje natural de todas las especies involucradas, conteniendo toda la variación identificada en los pliegos seleccionados, y una clave interactiva
(Intkey_taxones) para discriminar entre especies y facilitar las actividades de determinación.

\section{Resultados y Discusión}

\section{Nuevos caracteres observados en} las especies venezolanas de Merremia con potencial taxonómico

En función de las observaciones realizadas sobre los pliegos de los herbarios y en comparación con lo reportado por la literatura, se logró identificar un conjunto de caracteres referidos al indumento, la partición de la lámina foliar, tipología de las inflorescencias y otros relacionados con el fruto, los cuales presentan importancia taxonómica para el grupo de especies evaluadas por la información potencial que de ellos se ha derivado. Los caracteres del indumento identificados en los especímenes de Merremia constituyeron el grupo más informativo y de mayor aplicación taxonómica dada la posibilidad de establecer diferencias entre las especies a partir de pliegos en estado vegetativo. En virtud de la variación observada para el indumento, se establecieron nueve caracteres principales relacionados con la presencia/ausencia de indumento en la estructura evaluada, el tipo de tricoma, color, orientación, distribución y cobertura, longitud del tricoma simple y número de brazos del tricoma estrellado. Los tricomas observados fueron filiformes, tuberculados, glandulares y estrellados (Fig. 1).

De acuerdo con las observaciones de los pliegos y la evaluación preliminar de los caracteres de la matriz aplicando la acción "Summary", se determinó un nuevo carácter denominado: cobertura del indumento. Este carácter está basado en la densidad de tricomas presentes en la superficie de la epidermis y se establecieron tres estados, a saber: cobertura laxa,
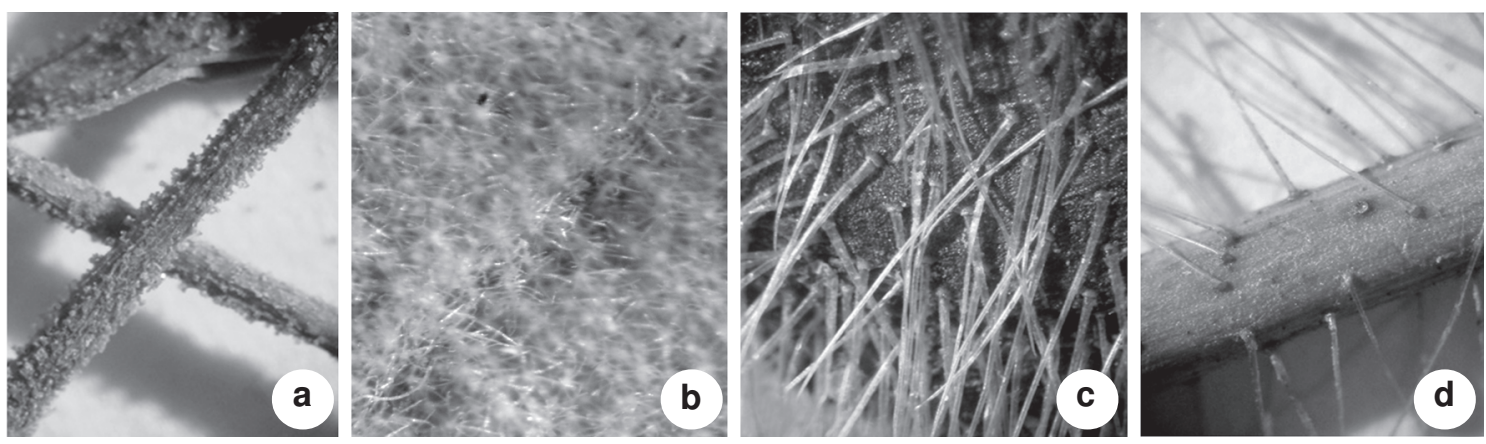

Figura 1 - Tipos de tricomas identificados en especies de Merremia: a. glandulares; b. estrellados; c. tuberculados; d. filiformes.

Figure 1 - Trichome types identified in Merremia species. a. glandular; b. stellate; c. tuberculated; d. filiforms. 
cuando la superficie observada sin tricomas es relativamente mayor a 50\%; cobertura medianamente densa, cuando sea menor a 50\%; y cobertura densa, cuando la superficie esté completamente oculta bajo los tricomas (Fig. 2).

Otros caracteres informativos observados en Merremia fueron la torción de las anteras durante la antesis y la acrescencia de los sépalos persistentes durante la fructificación conjuntamente con la posición de los mismos con respecto al eje longitudinal del fruto. Tal como destacan O'Donell (1941) y Ooststroom \& van Hoogland (1953), se observó la torción de las anteras de forma helicoidal en algunos taxones (Fig. 3a-b).

O'Donell (1941), Ooststroom \& van Hoogland (1953) y Austin (1982) han reportado la persistencia de los sépalos durante la fructificación en algunas especies de Merremia. Estos autores también señalan que el crecimiento en tamaño de estas estructuras, mejor conocido como acrescencia, es un carácter discutible y variable. Sin embargo, la posición y forma de los sépalos persistentes y acrescentes en los frutos de Merremia pueden ser utilizadas fácilmente para diferenciar especies. Comúnmente, los sépalos son paralelos al eje longitudinal del fruto, pero en pocos casos llegan a ser perpendiculares (patentes), como en $M$. cisoides (Lam.) Hallier f., M. dissecta (Jacq.) Hallier f. y $M$. macrocalyx (Ruiz \& Pav.) O'Donell (Fig. 3c-d).

\section{Descripciones en lenguaje natural}

Entre los principales productos del programa DELTA (Dallwitz \& Paine 1993) se encuentran las descripciones en lenguaje natural, ampliamente utilizadas con fines taxonómicos. Esta aplicación del sistema permitió redactar, en un sentido gramatical bastante preciso, las descripciones de los taxones señalando los estados observados en cada carácter y registrando la variación observada de forma más completa. Es necesario hacer notar que las descripciones resultantes fueron editadas para obtener una mejor expresión y sintaxis.

Para determinar la frecuencia de los estados de cada carácter, se recurrió a la acción "Summary" de la clave interactiva IntKey (Dallwitz \& Paine 1993) y se estableció una categorización de términos para explicar la variación de manera más concreta en función del número de veces que se registró el estado de un carácter respecto a la variación total observada para el mismo. Las categorías obtenidas se
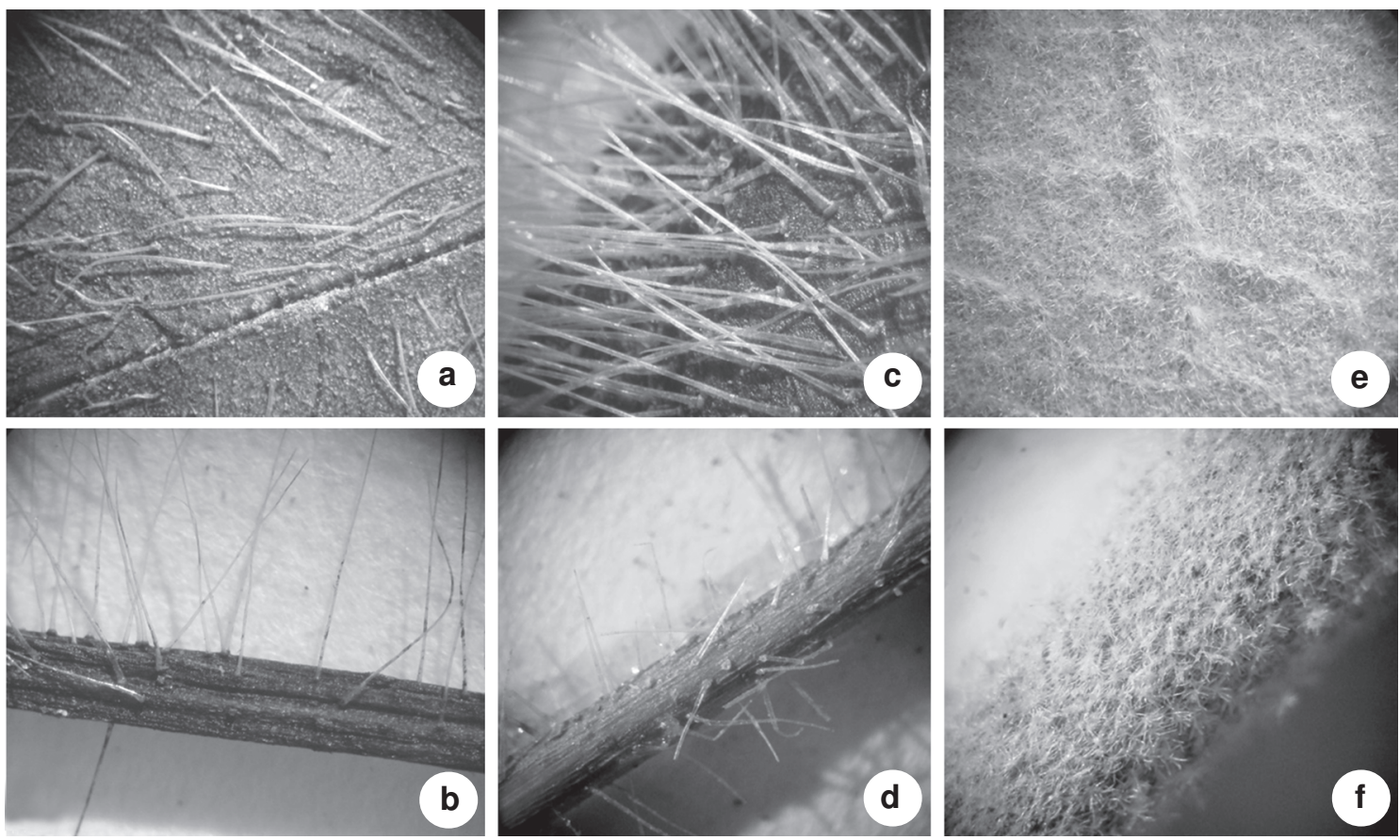

Figura 2 - Estados de la cobertura del indumento en estructuras planas y cilíndricas identificados en Merremia: a-b. cobertura laxa; c-d. cobertura medianamente densa; e-f. cobertura densa.

Figure 2 - States of indumentum cover in plain and cylindrical structures observed in Merremia: a-b. lax cover; c-d. moderately dense cover; e-f. dense cover. 


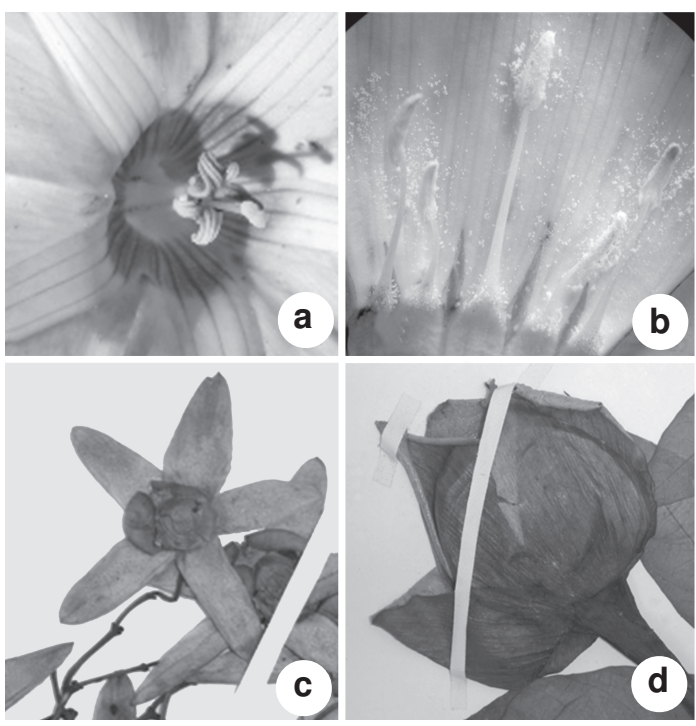

Figura 3 - a-b. Anteras durante la antesis: a. anteras torcidas (M. dissecta); b. anteras sin torción (M. tuberosa). c-d. Frutos de Merremia: c. sépalos perpendiculares (M. macrocalyx); d. sépalos paralelos (M. tuberosa).

Figure $3-\mathrm{a}-\mathrm{b}$. Anthers during anthesis: a. twisted anthers $(M$. dissecta); b. erect anthers (M. tuberosa). c-d. Fruits in Merremia: c. perpendicular sepals in fruit (M. macrocalyx); d.- parallel sepals (M. tuberosa)

expresaron de la siguiente manera: "raras veces", cuando la frecuencia del estado fue menor o igual al $10 \%$ de la variación registrada, "pocas veces/algunas veces" (20-25\%), "comúnmente/frecuentemente" (>80\%), "y/o" cuando las frecuencias se encontraron relativamente equivalentes (45-65\%).

Aplicando la matriz de caracteres en el Editor DELTA señalada en el anexo, se obtuvieron descripciones en lenguaje natural para cada taxón como en el ejemplo que sigue:

Sufrútices, volubles, tallos teretes, $1,3-3 \mathrm{~mm}$ diám.; lisos o ligeramente costulados. Hojas simples, enteras; pecíolos teretes, 8,3-110×0,4-7 mm. Láminas foliares ovadas, concoloras, de 2,87-14 × 0,84-11,9 $\mathrm{cm}$, base auriculada, raras veces cordada, ángulo obtuso o agudo, margen entero, ápice frecuentemente acuminado, ocasionalmente agudo o atenuado, con mucrones, nerviación actinódroma. Indumento: Tallos y pecíolos con tricomas filiformes, blancos, raras veces amarillentos, torcidos, adpresos o erectos, cobertura densa, medianamente densa a laxa, distribuidos en toda la extensión o algunas veces en el tercio apical. Láminas foliares pubescentes, en ocasiones glabras, tricomas frecuentemente distribuidos en toda la superficie en ambas caras, de cobertura medianamente densa en la cara abaxial. Inflorescencias en cimas umbeliformes, raras veces reducidas, 1-31 flores, frecuentemente 3-4 grados de ramificación, 6,6-140 $\mathrm{mm}$; pedúnculo terete, raras veces aplanado, 37-110 $\times 0,6-18 \mathrm{~mm}$, no ensanchado en la base, con sección; pedúnculos y pedicelos pubescentes, algunas veces glabros, tricomas en toda la superficie, blancos, raras veces translúcidos o amarillentos, filiformes, torcidos, adpresos o ascendentes o erectos, cobertura medianamente densa, pocas veces densa, raras veces laxa; brácteas y bractéolas caducas, raras veces persistentes, ovadas, raras veces elípticas; flores 1,8$3 \mathrm{~cm}$; pedicelos teretes, raras veces aplanados, $45 \times$ 0,7-1 mm; cáliz de 4-5 sépalos, glabros, raras veces pubescentes, tricomas filiformes, blancos, erectos, con cobertura laxa, distribuidos en el tercio medio, libres entre sí, con prefloración imbricada, obovados, raras veces ovados, obtusos en la base, margen entero con consistencia membranácea, cóncavos, ápice frecuentemente obtuso, pocas veces redondeado o acuminado, con mucrones; sépalo más externo de 7,5$11 \times 35-59 \mathrm{~mm}$; sépalo más interno de 7,5 ×5 mm, nervaduras inconspicuas; corola con frecuencia de 5 pétalos, algunas veces con 4 , con prefloración contorta o imbricada, amarilla, pubescentes, pocas veces glabra, tricomas filiformes torcidos, blancos, distribuidos en el tercio apical, en la zona interpetalar, con cobertura laxa o medianamente densa margen entero; bandas mediapetalinas concoloras, raras veces discoloras; lóbulos ovados a obovados, con margen entero, ápice obtuso, raras veces redondeado, frecuentemente mucronados, de 1-3,4 mm ×3-9 mm; tubo de la corola de 1-2,6 0 0,57-0,9 cm; limbo de 12,3-23,7 mm diám., nervaduras conspicuas, raras veces inconspicuas; androceo de 5 estambres, adnatos a la corola, libres entre sí, inclusos, insertos en el tercio superior de la corola, raras veces en el inferior, isodínamos o didínamos; filamentos de 2-5 $\mathrm{mm}$, glabros o con tricomas distribuidos en el tercio basal; anteras ovadas o triangulares, raras veces elípticas, 3-4 mm $\times 1 \mathrm{~mm}$, dorsifijas, con dehiscencia extrorsa, no torcidas en la antesis; gineceo sincárpico, con 2 lóculos, disco anular; ovario globoso, glabro, estigmas 2, frecuentemente esféricos, placentación central libre, con 2 óvulos por lóculo. Fruto dehiscente, globoso, 8-13 mm diam., superficie del exocarpo lisa; sépalos persistentes y acrescentes en el fruto; sépalo acrescente más externo 7-12,3 ×6,3-18 mm; sépalo acrescente más interno 6-11,7 $\mathrm{mm} \times 5-7 \mathrm{~mm}$, paralelos al eje, con 1 septo, raras veces 2 , de dehiscencia septicida, 2 lóculos; semillas trígonas, 2-4 por fruto, de 4-9,4 $\mathrm{mm}$, con tricomas filiformes, marrones, en toda la superficie. 


\section{Claves interactivas (IntKey)}

Una vez concretada la matriz de taxones y la clave interactiva resultante para taxones (Intkey_taxones) se ejecutaron diferentes acciones que ayudaron a determinar la circunscripción taxonómica de las entidades evaluadas, empleando la herramienta "Summary". Por medio de estas claves se obtuvieron diferencias y similitudes entre taxones, así como diagnósticos y descripciones de cada taxón.

\section{Análisis de agrupamiento de las} especies: relaciones y afinidades taxonómicas, morfológicas, ecológicas

A partir del análisis fenético con PAST (Hammer et al. 2001), se alcanzaron resultados provechosos utilizando los métodos UPGMA y de Ward. Los dendrogramas obtenidos muestran alta afinidad y clara definición entre los grupos de especímenes de los taxones evaluados. Se distinguen diferencias de agrupamiento sólo para dos taxones (M. wurdackii y M. cissoides) en el dendrograma por UPGMA (Fig. 4) y uno por Ward (M. wurdackii) (Fig. 5), ocasionadas principalmente por la falta de completitud de información en algunos pliegos ingresados. En el caso de $M$. wurdackii, sólo se codificaron datos de un único pliego depositado en VEN (wur_3) y los items señalados como wur_113 y wur_119 están referidos a la descripción y una fotografía del tipo, respectivamente. Esta misma topología en el agrupamiento se observa en el dendrograma obtenido por el método de Ward. La separación de $M$. cissoides dentro del dendrograma de la Fig. 4 (UPGMA) permitió identificar dos grupos formados por los pliegos en floración (cis_99cis_125-cis_121) y en estado vegetativo o en fructificación (cis_98-cis_100-cis_101).

También se observó que los grupos de especies trifolioladas ( $M$. nervosa Pittier y $M$. ternifoliola Pittier) se encuentran próximas entre sí en el dendrograma por UPGMA. Cabe destacar que en ambos dendrogramas, $M$. cissoides y $M$. quinquefolia (L.) Hallier f. aparecen completamente separadas entre sí, lo cual corrobora su posición como entidades completamente distintas, a pesar de

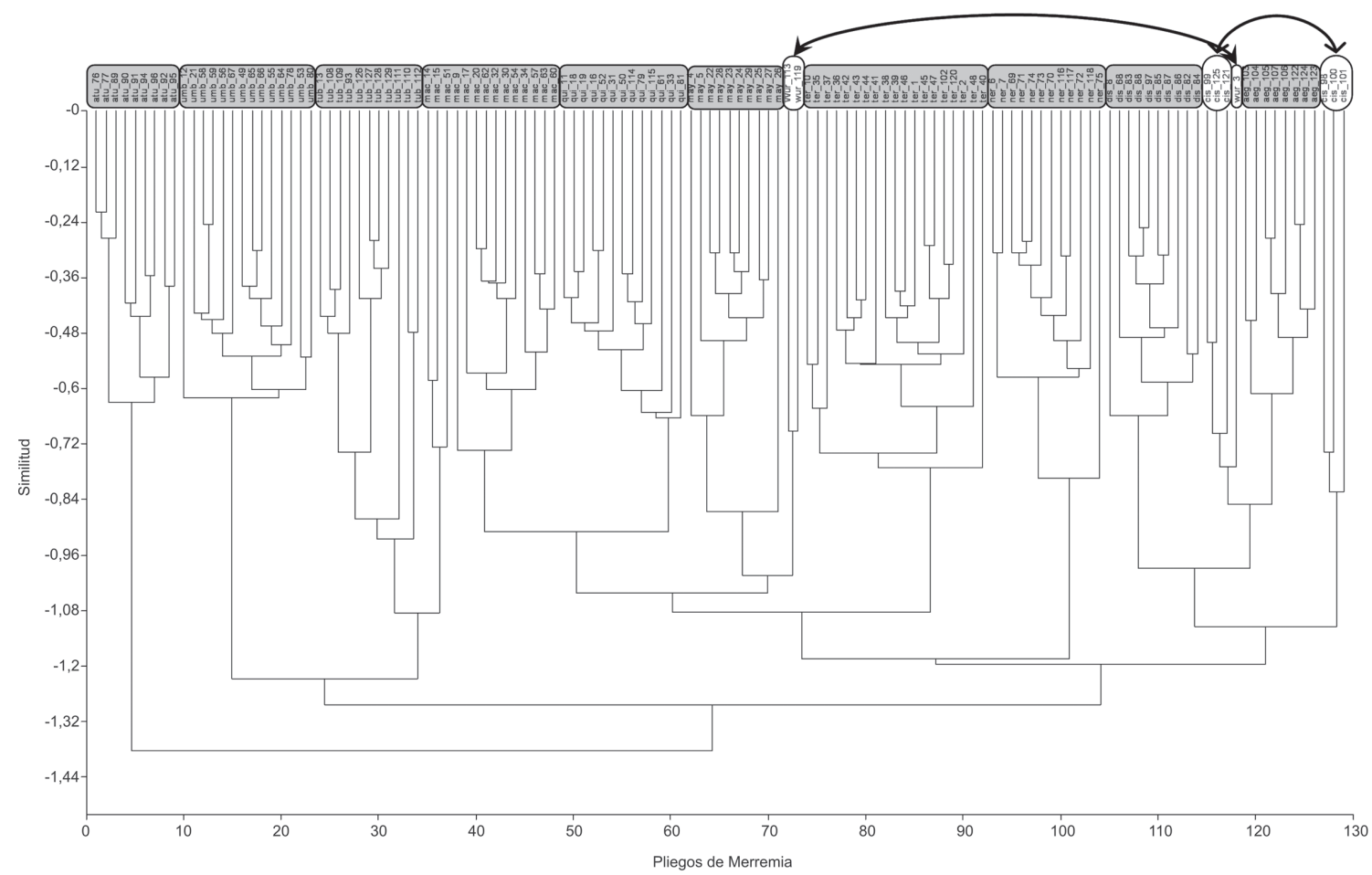

Figura 4 - Agrupamiento por similitud de los especímenes revisados de Merremia utilizando el método UPGMA con distancia euclideana. Las líneas arqueadas indican la disyunción de pliegos del mismo taxón dentro del agrupamiento enmarcados en rectángulos blancos.

Figure 4 - Similarity clustering of Merremia specimens using UPGMA with euclidean distance. Arched lines show disjunctive sheets from the same taxon marked in white rectangles. 


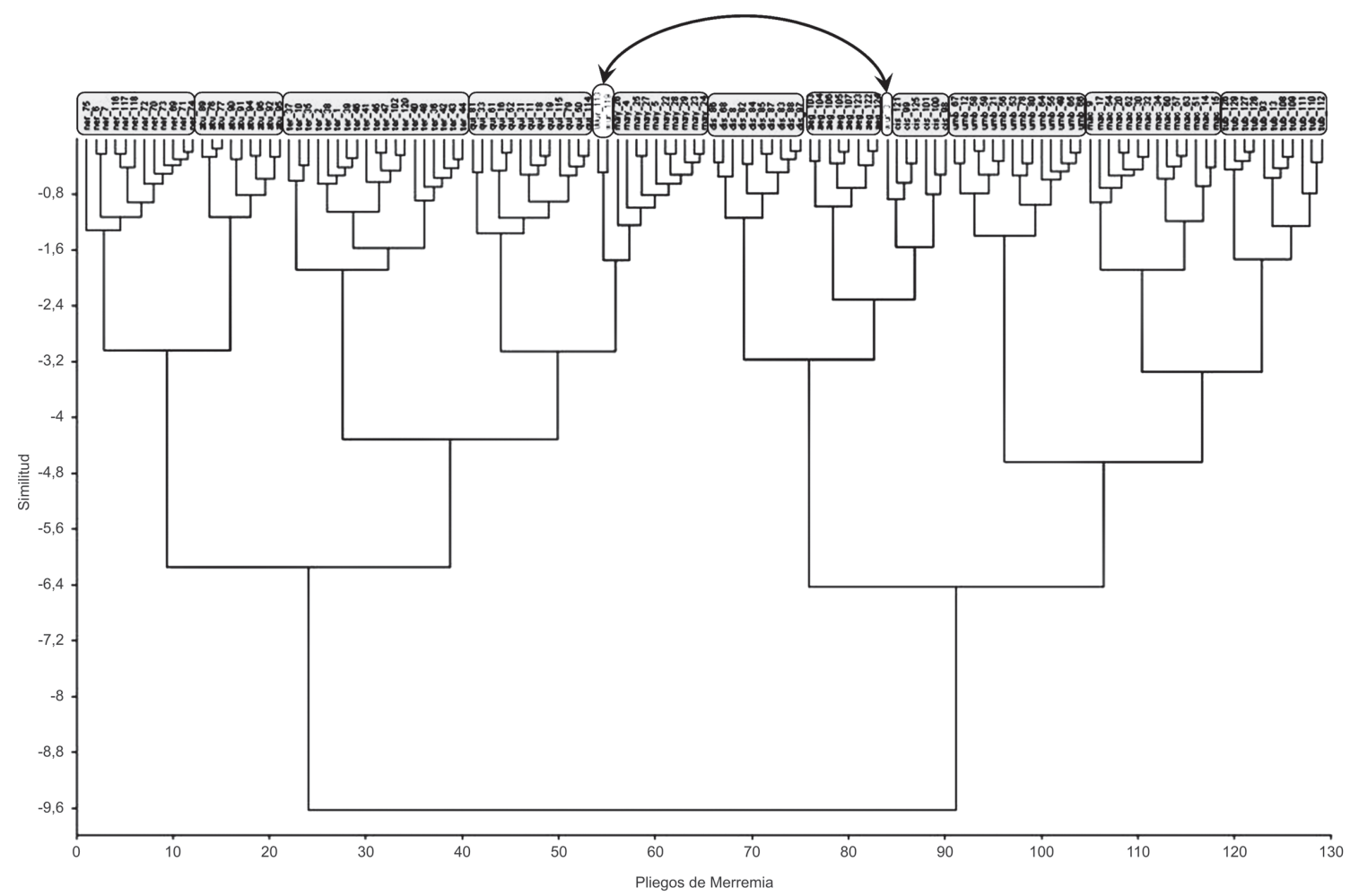

Figura 5 - Agrupamiento por similitud de los especímenes revisados de Merremia utilizando el método de Ward con distancia euclideana. Las líneas arqueadas indican la disyunción de pliegos del mismo taxón dentro del agrupamiento enmarcados en rectángulos blancos.

Figure 5 - Similarity clustering of Merremia specimens using Ward with euclidean distance. Arched lines show disjunctive sheets from the same taxon marked in white rectangles.

su apariencia tan similar, y comúnmente se distingue la alta afinidad de $M$. cissoides con $M$. aegyptia (L.) Urb. y M. dissecta.

\section{Consideraciones Finales}

La aplicación de una herramienta computacional como DELTA ha demostrado la obtención de resultados útiles para estudios con fines taxonómicos gracias a su flexibilidad y practicidad, además de su eficiencia en el manejo de gran cantidad de datos. Con este programa, se estableció un tiempo de trabajo de tres meses para el ingreso de los datos a partir de los pliegos y tres meses adicionales para la consolidación de la matriz, edición de caracteres, ejecución de los análisis estadísticos y preparación de las descripciones.

Se consolidó una matriz de 209 caracteres conformada por 146 caracteres cualitativos binarios y multiestados, 60 caracteres cuantitativos y tres caracteres de texto. La matriz de caracteres y estados del Editor DELTA y la clave interactiva de los taxones pueden ser solicitadas al autor por correo electrónico. Los resultados obtenidos corroboran la presencia de 12 especies de Merremia para Venezuela, de las cuales, $M$. wurdackii, que fue descrita recientemente, no había sido tomada en cuenta por el trabajo de Austin (1982), y que posteriormente reporta Tapias (2008). Originalmente, aunque no fue mencionado por Austin (1982) para Venezuela y otros países, que existían problemas para distinguir $M$. cissoides de $M$. quinquefolia, basados en material vegetativo. Como se ha mencionado previamente, DELTA permitió identificar aquellos caracteres de mayor estabilidad y utilidad, y separar claramente estas dos especies al establecer los límites de su circunscripción taxonómica. Por tanto, estos taxones pueden ser exitosamente diferenciados entre sí, gracias a la aplicación de claves interactivas. Adicionalmente, el uso de la herramienta DELTA permitió la preparación de las descripciones más completas de los taxones, tomando en cuenta los rangos morfológicos en función del gran número de especímenes examinados. 
Esto es una aporte significativo, con respecto al trabajo inicial realizado en el país por Austin (1982) y que ahora se ve adelantado en el trabajo conducido sobre el género Merremia por Ferrer-Pereira et al. (2010) en el siguiente artículo de esta revista.

\section{Agradecimientos}

A la Fundación Instituto Botánico de Venezuela, especialmente a su presidente Aníbal Castillo-Suárez por el financiamiento y apoyo en la logística; al Herbario Nacional de Venezuela por poner a disposición su base de datos; a los curadores de MY, PORT, GUYN y VEN por los préstamos de los pliegos. Este trabajo es producto de la asignatura Herramientas Computacionales para el Análisis Taxonómico, del Postgrado en Botánica (Instituto de Biología Experimental, UCV) y del Postgrado en Botánica y Taxonomía Neotropical (ULA).

\section{REFERENCIAS}

Austin, D.F. 1982. Convolvulaceae. In: Luces de Febres, Z. \& Steyermark (eds.). Flora de Venezuela 8(3). Ediciones Fundación Educación Ambiental, Caracas. Pp. 15-226.

Austin, D.F. \& Staples, G.W. 1981. Convolvulaceae. In: Maguire, B. (ed.). The botany of the Guayana highlands Part XI. Vol. 32. Memoires of the New York Botanical Garden. Pp. 309-323.

Austin, D.F. \& Staples, G.W. 1983. Additions and changes in the neotropical Convolvulaceae: notes on Merremia, Operculina, and Turbina. Journal of the Arnold Arboretum 64: 483-489.

Clayton, W.D.; Harman, K.T. \& Williamson, H. 2010. GrassBase - The Online World Grass Flora. The Board of Trustees, Royal Botanic Gardens, Kew. Disponible en <http://www.kew.org/data/grassbase/ index.html>. Acceso en 05 Julio 2010.

Dallwitz, M.J. \& Paine, T.A. 1993 onwards. DELTA DEscription Language for TAxonomy. Version 1.04 (for Windows). CSIRO, Division of Entomology, Canberra. Disponible en <http://delta-intkey.com>. Acceso en 15 Octubre 2008.

Dallwitz, M.J.; Paine, T.A. \& Zurcher, E.J. 2008. IntKey The interactive key program. V. 5.11 (for Windows). CSIRO. Division of Entomology. Canberra. Disponible en <http://delta-intkey.com>. Acceso en 15 Octubre 2008.

Dilcher, D.L. 1974. Approaches to the identification of angiosperm leaf remains. The Botanical Review 40: 1-157.

Estrada, J. 1995. Cordia subgénero Varronia (Boraginaceae). In: Díaz P.S.; Telleria, M.T.; Castroviejo, S.; JaramilloM., W.; Ruíz-C., P. \& Lozano-C. (eds.). Flora de Colombia 14: 1-74.
Ferrer-Pereira, H.E.; Vivas-Arroyo, Y.; Nozawa, S.; Hokche, O.; Pérez-Cortéz, S.; Rodríguez, L.; Mostacero, J. \& Estrada, J. 2010. El género Merremia (Convolvulaceae) en Venezuela. Rodriguésia 61: 639-660.

Garbiso, C. \& Estrada, J. 2001. Sinopsis taxonómica de Bomarea Mirb. subgénero Bomarea (Alstroemeriaceae) para Venezuela. PlantULA 3: 11-39.

Hammer, Ø.; Harper, D.A.T. \& Ryan, P.D. 2001. PAST: Paleontological statistics software package for education and data analysis (v. 1.88 for Windows). Palaeontologia Electronica 4: 1-9. Disponible en $<$ http://palaeo-electronica.org/2001_1/past/ issue1_01.htm>. Acceso 25 Febrero 2009.

Hickey, L. 1973. Classification of the architecture of dicotyledonous leaves. American Journal of Botany 60: 17-33.

O`Donell, C.A. 1941. Revisión de las especies americanas de Merremia (Convolvulaceae). Lilloa 6: 467-554.

Ooststroom, S.J. \& van Hoogland, R.D. 1953. Convolvulaceae. In: van Steenis, C.G.G.J.(ed.). Flora Malesiana. Vol. 1. Pp. 388-512.

Payne, W.W. 1978. A glossary of plant hair terminology. Brittonia 30: 239-255.

Pittier, H.F.; Badillo Franceri, V.M.; Lasser, T.; Schnee, L. \&Luces Febres, Z. 1945. Catálogo de la flora venezolana. Litografía y Tipografía Vargas, Caracas. 576p.

Poe, S. \& Wiens, J.J. 2000. Character selection and the methodology of morphological phylogenetics. In: Wiens, J.J. (ed.). Phylogenetic analysis of morphological data. Smithsonian Institution Press, Washington. Pp. 26-30.

Radford, A.E.; Dickinson, W.C.; Massey, J.R. \& Bell, C.R. 1967. Vascular plant systematics. Harper \& Row, Harvard. 891p.

Sokal, R.R. \& Sneath, P.H.A. 1973. Numerical taxonomy. Freeman, San Francisco. 573p.

Stefanovic, S.; Krueger, L. \& Olmstead, R.G. 2002. Monophyly of the Convolvulaceae and circumscription of their major lineages based on DNA sequences of multiple chloroplast loci. American Journal of Botany 89: 1510-1522.

Stefanovic, S.; Austin, D.F. \& Olmstead, R.G. 2003. Classification of Convolvulaceae: a phylogenetic approach. Systematic Botany 28: 791-806.

Tapias, R. 2008. Convovulaceae. In: Hokche, O.; Berry, P. \& Huber, O. (eds.). Nuevo catálogo de la flora vascular de Venezuela. Fundación Instituto Botánico de Venezuela, Caracas. Pp. 339-344.

Ward, J.H. 1963. Hierarchical groupings to optimize an objective function. Journal of the American Statistical Association 58: 234-244.

Weberling, F. 1996. Morphology of flowers and inflorescences. Cambridge University Press, Cambridge. 404p. 